\title{
Mutation Induction, Detection and Breeding to Resist Viral and Fungal Diseases and Thermal Extremism for Vegetables
}

\author{
Yousif DP* and Al Janabi AA \\ Agriculture Research Directorate, Ministry of Science and Technology, Iraq
}

${ }^{*}$ Corresponding author: Yousif DP, Agriculture Research Directorate, Ministry of Science and Technology, Iraq.

Received Date: January 11, 2020

Published Date: January 21, 2020

\section{Opinion}

To artificially induce hereditary changes in plants, either physical or chemical agents are used. Ionizing radiation is a widely used physical agent to treat the seeds and other crops plant material to create heritable mutations. On other means, one of the most important breakthroughs in the history of genetics was the discovery that mutations can be induced by physical and chemical mutagens (agents that change the genetic base of an organism). Mutation induction, together with mutation detection a key element of mutation breeding, has been an important tool for plant breeders for more than 70 years to increase the genetic diversity of plants and derive new mutant lines with improved characteristics.

Generally, mutations are a result of large-scale deletions, inversions or translocations of chromosomes, or from point mutations (a type of mutation that causes a single change, insertion or deletion of the genetic material) in the DNA. Physical mutagens most often result in chromosome changes and larger DNA deletions while mutagenic chemicals typically cause point mutations. The degree of mutation also depends on the tissue and the time and dosage of exposure. DNA mutations are generally of the most interest to breeders.

Ionizing radiation, a Physical mutagen, mostly, can increase the natural mutation rate by 1,000 to 1 million-fold, and commonly used to induce heritable genetic changes. Since the 1960s, X-ray and gamma rays with a cobalt-60 source, have become the widely used mutagenic agents in plant mutation breeding.

The next step in mutation breeding is to detect which plants have indeed produced the desired new traits. The detection of novel induced mutants presents a major challenge because it occurs with low frequencies which requires the creation of very large mutant
M1 population. It is very important how-to asses and select the useful mutants that have developed a new desirable trait mutated plant.

Collaboration between the FAO and IAEA in joint projects results to develop and adopt nuclear-based technologies that optimize mutation induction practices, with the goal to intensify crop production and preserve natural resources.

Screening protocols, such as for salt and drought tolerance screening methods or disease screening protocols, are efficient methods and practical tool for mutant phenotyping detection and breeding. Recent detection technologies have increased the efficiency of identifying the DNA changes that generate a new trait. TILLING (Targeting Induced Local Lesions in Genomes), allows directed identification of mutations in a specific gene. Detection of novel induced mutations has long been a bottleneck in plant mutation breeding. Screening for desired traits in plants and section practices increased and accelerate the development of mutant lines into commercial varieties.

\section{Mutation Breeding}

Mutation breeding is the further step of mutation induction and mutation detection. It has many comparative advantages: it is cost effective, quick, proven and robust. It is also transferrable, ubiquitously applicable, non-hazardous and environmentally friendly. More than 3,200 mutant varieties - including numerous crops, ornamentals and trees - have officially been released for commercial use in more than 210 plant species from over 70 countries (FAO/IAEA Mutant Varieties Database).

Plant biotechnologies play an important role in mutation breeding. Plant tissue culture techniques are powerful tools in 
shortening the time needed to generate breeding mutant lines. This is a bottleneck for the exploitation of induced crop mutations that are recessive (in genetics, when one characteristic of a gene is not expressed because a more dominant one is displayed).

\section{Project planning}

Virus diseases became limited factor for vegetables production in Iraq. However, the virus host became available continuously and so causing high yield loss seasonally.

Tomato is an important crop which occupied an advanced degree in food demand all over the world and in Iraq is the first one of vegetables. There is many reasons consider a limited factor of this crop productivity. Virus infection especially Tomato Yellow Leaf Curl Virus (TYLCV) is in the present of the main problem and may lead to high or full losses of yield in some years. High temperature degree in summer which rise to about $45-50$ o causes reduce flowering and fail of setting and so lead to high yield losses.

Potato also severe of many devastating virus diseases causing high productivity reduction annually, which indicates the urgent needs to reproduce of virus free tuber continuously to avoid their continuous concentration excess with successive generations. The shortage of thermal moderation period $\left(20-25^{\circ}\right)$ which is suitable for potato growing, and tubers production beginning after one month of emergence in March to last of May in spring, and in October to half of December in Autumn cultivation.

Eggplant and pepper are important crops for food and health. Virus diseases such Eggplant and Pepper Mosaic became also a limited factor of production, in addition to the grey mold and white mold caused by the fungus Botrytis cinerae, and Sclerotinia sclerotiourum are important, especially in plastic houses. High temperature degree in summer causing to slow plant development, fruit growth, flower falling, and weak setting.

Searching for varieties or variants having resistance, or moderately resistance, or tolerance, or slow virus or fungus disease development became of high importance to avoid the negative impact of these diseases. Irradiation of seeds or tuber buds and plant tissue culture are of the important ways to induce genetic variation and developing short plant growth period or suitable for thermal extremism. This project including the following main subjects which presents the target project objectives:

- Determining of the mainly devastating virus and identification of their virulent strains.

- $\quad$ Testing of all available varieties of each crop to its important viruses' strains and determines their different level of resistance, or tolerance, or slow symptoms development.

- Irradiation of seeds, or tubers, or tissue cultures of the selected varieties to induce new resistant traits and/ or new mutant cultivars.

\section{Acknowledgment}

None.

\section{Conflict of Interest}

No conflict of interest. 